\title{
Lurasidone in the treatment of schizophrenia: a 6-week, placebo-controlled study
}

\author{
Masaaki Ogasa • Tatsuya Kimura • \\ Mitsutaka Nakamura • John Guarino
}

Received: 14 May 2012 / Accepted: 30 July 2012 /Published online: 19 August 2012

(C) The Author(s) 2012. This article is published with open access at Springerlink.com

\begin{abstract}
Rationale There is an unmet need in the treatment of schizophrenia for effective medications with fewer adverse effects.

Objective This study aims to evaluate the efficacy and safety of lurasidone, an atypical antipsychotic, for the treatment of schizophrenia.

Methods Patients with an acute exacerbation of schizophrenia were randomized to 6 weeks of double-blind treatment with once-daily, fixed-dose lurasidone $40 \mathrm{mg}(N=50)$, lurasidone $120 \mathrm{mg}(N=49)$, or placebo $(N=50)$. The primary efficacy measure was mean change from baseline to day 42 (last observation carried forward) in the Brief Psychiatric Rating Scale derived (BPRSd) from the Positive and Negative Syndrome Scale (PANSS).

Results Mean change in BPRSd was significantly greater in patients receiving lurasidone 40 and $120 \mathrm{mg}$ /day versus placebo $(-9.4$ and -11.0 versus $-3.8 ; p=0.018$ and 0.004 , respectively). Treatment with lurasidone $120 \mathrm{mg}$ /day was superior to placebo across all secondary measures, including PANSS total $(p=0.009)$, PANSS positive $(p=0.005)$, PANSS
\end{abstract}

\footnotetext{
M. Ogasa $(\bowtie) \cdot$ T. Kimura

Dainippon Sumitomo Pharma Co., Ltd., 6-8, Doshomachi 2-Chome, Chuo-ku,

Osaka 541-0045, Japan

e-mail: masaaki-ogasa@ds-pharma.co.jp

M. Ogasa

Kanazawa University,

Kanazawa, Ishikawa, Japan

M. Nakamura

Setsunan University,

Neyagawa, Osaka, Japan

J. Guarino

Sunovion Pharmaceuticals, Inc.,

Fort Lee, NJ, USA
}

negative $(p=0.011)$, and PANSS general psychopathology $(p=0.023)$ subscales and Clinical Global Impression of Severity (CGI-S; $p=0.001$ ). Treatment with lurasidone $40 \mathrm{mg} /$ day was superior to placebo on the PANSS positive subscale $(p=0.018)$ and CGI-S $(p=0.002)$. The most common adverse events for patients receiving lurasidone were nausea $(16.2$ versus $4.0 \%$ for placebo) and sedation (16.2 versus $10.0 \%$ for placebo). Minimal changes in weight, cholesterol, triglyceride, and glucose levels were observed.

Conclusions In this study, which was limited by a relatively high discontinuation rate, lurasidone provided effective treatment for patients with acute exacerbation of chronic schizophrenia and had minimal effects on weight and metabolic parameters.

Keywords Lurasidone $\cdot$ Schizophrenia $\cdot$ Atypical antipsychotics $\cdot$ Drug therapy $\cdot$ Clinical trial

\section{Introduction}

Schizophrenia is a chronic and debilitating psychiatric disorder that affects an estimated $1 \%$ of the adult population (Regier et al. 1993). Although improvement is noted over time for some patients, the majority of patients experience at least some persisting symptoms despite treatment (Regier et al. 1993). The annual cost of schizophrenia in the USA is estimated as $\$ 63$ billion with about $\$ 23$ billion attributable to direct healthcare costs (Wu et al. 2005).

Although first-generation or conventional antipsychotic medications produce improvement in the positive symptoms of schizophrenia, they often result in serious adverse effects, including extrapyramidal symptoms (EPS) (Schotte et al. 1996). Conventional antipsychotic medications have been superseded by second-generation or atypical antipsychotics, which are now the mainstay of pharmacotherapy for schizophrenia (Sernyak and Rosenheck 2008). These newer 
agents, which are antagonists at dopamine $\mathrm{D}_{2}$ and 5-hydroxytryptamine $2 \mathrm{~A}\left(5-\mathrm{HT}_{2 \mathrm{~A}}\right)$ receptors, have demonstrated antipsychotic efficacy and are generally associated with a lower propensity for EPS than conventional antipsychotics (Leucht et al. 2009a; Meltzer et al. 2003; Schotte et al. 1996).

Most atypical antipsychotics display relatively high affinity for $\alpha_{1}$ adrenergic receptors, muscarinic receptors, and $\mathrm{H}_{1}$ histaminergic receptors (Ishibashi et al. 2010; Kroeze et al. 2003). The association of some atypical antipsychotics with sedation, impairment in cognitive function, and weight gain is thought to be due in part to effects at these receptors (Ishibashi et al. 2010; Kroeze et al. 2003). In addition, some atypical antipsychotics produce unfavorable changes in plasma lipid levels and glucose metabolism (American Diabetes Association et al. 2004; Henderson et al. 2005; Newcomer 2007), and there is substantial variability among atypical antipsychotics in the magnitude of weight gain and metabolic changes resulting from treatment (Newcomer 2007).

The occurrence of adverse effects can negatively affect treatment adherence. Results of a large, randomized, double-blind study sponsored by the National Institute of Mental Health showed that approximately 65 to $80 \%$ of outpatients with chronic schizophrenia discontinue their antipsychotic medications, often because of lack of efficacy or intolerable adverse effects (Lieberman et al. 2005). The most common reasons for discontinuation because of intolerability were weight gain or metabolic effects, EPS, and sedation. These observations underscore a need for additional treatment options for patients with schizophrenia.

Lurasidone is an atypical antipsychotic that was approved by the US Food and Drug Administration (FDA) in October 2010 for the treatment of patients with schizophrenia (Latuda ${ }^{\circledR}$ 2010). In vitro receptor binding studies and preclinical behavioral studies indicate that lurasidone is an antagonist with high affinity at $\mathrm{D}_{2}, 5-\mathrm{HT}_{2 \mathrm{~A}}$, and $5-\mathrm{HT}_{7}$ receptors; moderate affinity at human $\alpha_{2 \mathrm{C}}$ adrenergic receptors; and weak affinity at $\alpha_{2 \mathrm{~A}}$ adrenergic receptors (Ishibashi et al. 2010). In addition, lurasidone acts as a partial agonist at $5-\mathrm{HT}_{1 \mathrm{~A}}$ receptors, with moderate to high affinity. Notably, lurasidone exhibits no appreciable affinity for histamine $\mathrm{H}_{1}$ and muscarinic $\mathrm{M}_{1}$ receptors (Ishibashi et al. 2010).

We report here the findings of the first in a series of short-term, placebo-controlled studies conducted to assess the efficacy and safety of lurasidone for the treatment of schizophrenia (Nakamura et al. 2009; Meltzer et al. 2011). This 6-week, randomized, double-blind, placebo-controlled, phase 2 study evaluated the efficacy and safety of two fixed, daily doses of lurasidone ( 40 and $120 \mathrm{mg}$ ) for patients hospitalized for an acute exacerbation of schizophrenia.

\section{Methods}

\section{Patients}

The study enrolled men and women between 18 and 64 years of age who met Diagnostic and Statistical Manual of Mental Disorders, 4th Edition (DSM-IV) criteria for primary diagnosis of schizophrenia as established by the Structured Clinical Interview for DSM-IV Disorders-Clinician's Version (SCID-CV) (First et al. 1997) and were hospitalized for an acute exacerbation of symptoms. Patients were also required to have illness duration of at least 1 year, no psychiatric hospitalization within the 3 months prior to study entry, a Brief Psychiatric Rating Scale score derived (BPRSd) from the Positive and Negative Syndrome Scale (PANSS) of $\geq 42$ (Guy 1976; Kay et al. 1987), a score of $\geq 4$ on two or more items of the positive symptoms subscale on the PANSS, and a Clinical Global Impression of Severity (CGI-S) score of $\geq 4$ (moderate) (Guy 1976). Patients were excluded if they had an acute or unstable medical condition; a DSM-IV diagnosis of schizophreniform disorder, schizoaffective disorder, or the catatonic or residual types of schizophrenia; evidence of another chronic central nervous system disorder; or an existing movement disorder. Patients were also excluded if they had a history of resistance to treatment with neuroleptics, defined as failure to respond to two or more antipsychotic agents from two different classes, or clozapine, administered at adequate doses for sufficient duration, or had received depot antipsychotics within one standard treatment cycle.

All patients provided written informed consent prior to study enrollment. The study protocol and all related forms and amendments were approved by an independent ethics committee associated with each study center. The study was conducted in accordance with FDA guidance documents on Good Clinical Practice, as well as the International Conference on Harmonisation guidelines on Good Clinical Practices (1996) and the ethical principles of the 1964 Declaration of Helsinki.

Study design and treatment

This was a 6-week, multicenter, randomized, fixed-dose, double-blind, parallel-group, placebo-controlled study conducted at 16 sites in the USA between February and December 2001. The study included three periods: a screening period of up to 14 days, a single-blind placebo washout period of up to 7 days, and a 6-week double-blind treatment period. Following the washout period, patients meeting entry criteria were randomized, in a 1:1:1 ratio, to oncedaily fixed doses of lurasidone $40 \mathrm{mg}$, lurasidone $120 \mathrm{mg}$, or placebo. Patients randomized to the $120-\mathrm{mg}$ /day lurasidone dose received $80 \mathrm{mg}$ on day 1 , and the dose was increased to 
$120 \mathrm{mg}$ by day 6 . All patients remained in the hospital during the screening and placebo washout periods and through the first 2 to 4 weeks of the double-blind treatment period. Patients with a Clinical Global Impression of Improvement (CGI-I) score of $\leq 4$ were then eligible for discharge from the hospital and continued study participation as outpatients. Patients were discontinued from the study if they did not show sufficient improvement to permit discharge from the hospital by week 4 of the double-blind treatment period.

Treatment compliance at each study visit was calculated as the number of tablets taken (i.e., the number of tablets no longer in the package) divided by the number of tablets that should have been taken, multiplied by 100 .

\section{Concomitant medications}

Benztropine mesylate or biperiden (1-2 mg twice daily) were permitted for the treatment of EPS; prophylactic administration of medications for EPS was prohibited. Lorazepam (up to $8 \mathrm{mg} /$ day), zolpidem (up to $10 \mathrm{mg}$ /day), temazepam (up to $30 \mathrm{mg} /$ day), or chloral hydrate (up to $1500 \mathrm{mg}$ /day) were used as rescue medication for symptom exacerbation. As-needed use of these concomitant medications was permitted for no more than five consecutive days during the double-blind period until 8 hours prior to efficacy assessments. Other psychotropic medications were prohibited.

\section{Assessments}

\section{Efficacy}

The primary efficacy measure was the mean change on the BPRSd score from baseline to week 6, assessed as the last observation carried forward (LOCF). The BPRSd comprises 18 items (derived from the PANSS) rated on a scale of $1=$ not present to $7=$ severe; therefore, the minimum possible score is 18 , and the maximum possible score is 126 . Secondary efficacy measures included mean change from baseline to week 6 (LOCF) on the PANSS total score, as well as PANSS positive symptoms, negative symptoms, and general psychopathology subscale scores (Lindenmayer et al. 1995); the CGI-S (Guy 1976); and the CGI-I. All efficacy assessments were completed at screening, at baseline, on days 3 and 7 , and weekly thereafter (or on early termination).

\section{Safety and tolerability}

All adverse events volunteered or observed during the study were recorded, together with their severity and duration. Assessment of movement disorders occurred at every study visit (or early termination) and included administration of the 10-item Simpson-Angus Scale (SAS) to evaluate parkinsonism $(0=$ normal to $4=$ most severe $)($ Simpson and Angus 1970), the 4-item Barnes Akathisia Scale (BAS) to evaluate akathisia ( $0=$ normal to $5=$ most severe) (Barnes 1989), and the 12-item Abnormal Involuntary Movement Scale (AIMS) to evaluate tardive dyskinesia $(0=$ normal to $4=$ most severe) (Guy 1976). Other safety measures included 12-lead ECG, weight, vital signs, and clinical laboratory assessments (hematology, serum chemistry, and urinalysis). When possible, laboratory specimens were collected with patients in the fasted state.

\section{Statistical analysis}

Allowing for a $10 \%$ dropout rate prior to the first efficacy assessment, it was estimated that 44 patients would be needed per group (i.e., 132 patients total) to detect a standardized treatment difference of 0.730 between the lurasidone and placebo groups at $90 \%$ power (two tailed) and at an alpha level of 0.050 (two sided). A $35 \%$ dropout rate during the washout period was assumed. Therefore, it was planned to enter approximately 205 patients into the placebo washout period to ensure that 132 patients were randomized into the study.

Efficacy analyses were performed using the intent-totreat (ITT) population, which consisted of all randomized patients who received at least one dose of study medication at the daily dose for the group (i.e., $40 \mathrm{mg}$ lurasidone, $120 \mathrm{mg}$ lurasidone, or placebo) and had at least one efficacy evaluation on or after day 3. Observed case analyses were also conducted for the primary and secondary efficacy measures, with completers represented by observed cases at day 42 . The safety population comprised all patients who received at least one dose of study medication and was used for all safety analyses.

Comparisons among treatment groups at baseline were performed using a one-way analysis of variance, with treatment as a term in the model. For the primary efficacy measure of change from baseline to week 6 (LOCF) on the BPRSd, an analysis of covariance (ANCOVA) model was used with effects for center, treatment, and center-by-treatment interaction, and baseline BPRSd as the covariate. If the center-by-treatment interaction was not significant $(p \geq 0.10)$, it was removed from the model. If the overall ANCOVA of the baseline-to-endpoint change score was significant, pairwise comparisons were performed using a Dunnett $t$ test. The type I error rate for rejecting a null hypothesis was set at 0.050 (two sided) for both the ANCOVA and the Dunnett test.

Secondary efficacy measures of change from baseline in the PANSS total and subscale scores, CGI-S, and CGI-I, 
were analyzed using ANCOVA models. The interaction term was excluded from the PANSS and CGI-I models.

Patients with a reduction of $\geq 20 \%$ from baseline in BPRSd or with a CGI-I score of 1 or 2 were classified as treatment responders. The proportion of responders versus nonresponders was compared using a Cochran-MantelHaenszel test controlling for center. Cohen's $d$ treatment effect sizes were calculated for primary and secondary efficacy measures as the difference in least-squares (LS) mean change score for lurasidone ( 40 or $120 \mathrm{mg} /$ day) and placebo (LOCF) divided by the pooled standard deviation.

For safety analyses, all statistical tests were two-sided at an alpha level of 0.05 . One-way ANCOVA was used to analyze changes from baseline in the composite (total score) SAS, BAS, and AIMS scores. The Fisher exact test was used for between-group comparisons of the rates of adverse events.

\section{Results}

Of 223 patients who were screened, 149 met entry criteria at the end of the washout period and were randomized to double-blind treatment (50 to lurasidone $40 \mathrm{mg} /$ day, 49 to lurasidone $120 \mathrm{mg} /$ day, and 50 to placebo) (Fig. 1). Baseline characteristics were similar across treatment groups (Table 1). The majority of patients in each group were men who met criteria for the paranoid subtype of schizophrenia. The ITT population, used for efficacy analyses, included 49 patients assigned to lurasidone $40 \mathrm{mg} /$ day, 47 assigned to lurasidone $120 \mathrm{mg} /$ day, and 49 assigned to placebo. All randomized patients were included in the safety population. Overall, 51 patients completed the study: 16 $(32.0 \%)$ in the $40 \mathrm{mg}$ /day group, $20(40.8 \%)$ in the $120 \mathrm{-mg} /$ day group, and $15(30.0 \%)$ in the placebo group. The most common reason for discontinuation was withdrawal of consent $(24.8 \%$ of randomized patients across all treatment groups) (Fig. 1). Lack of efficacy was the most common reason for discontinuation in the placebo group (32.0\%) and was cited less frequently as a reason for discontinuation in the 40 $(22.0 \%)$ and $120 \mathrm{mg}$ /day lurasidone $(12.2 \%)$ groups. The placebo group had the lowest rate of discontinuation due to adverse events $(4.0 \%)$, whereas rates were higher and similar for the $40(12.0 \%)$ and $120 \mathrm{mg} /$ day lurasidone $(12.2 \%)$ groups.

\section{Study treatment}

The mean (standard deviation) duration of exposure to study treatment was similar among patients randomized to lurasidone $40 \mathrm{mg}$ /day (23.4 (15.9) days), lurasidone
$120 \mathrm{mg}$ /day (23.0 (16.7) days), or placebo (22.2 (15.8) days). Mean treatment compliance was $97.5,99.5$, and $98.9 \%$ for the lurasidone $40 \mathrm{mg} /$ day, lurasidone $120 \mathrm{mg} /$ day, and placebo groups, respectively. The majority of patients received benzodiazepines at least once during the study: $90.0 \%$ of patients in the lurasidone $40 \mathrm{mg}$ /day group, $87.8 \%$ in the lurasidone $120 \mathrm{mg} /$ day group, and $82.0 \%$ in the placebo group. The mean daily dose of lorazepam was calculated by week and ranged from 0.5 to $1.3 \mathrm{mg}$ for patients receiving lurasidone $40 \mathrm{mg}$ /day, 0.9 to $1.7 \mathrm{mg}$ for patients receiving lurasidone $120 \mathrm{mg} / \mathrm{day}$, and 1.2 to $2.6 \mathrm{mg}$ for patients receiving placebo.

\section{Efficacy}

The LS mean change in BPRSd score (primary efficacy measure) from baseline to week 6 (LOCF) was significantly greater with lurasidone $40(-9.4 ; p=0.018$ versus placebo) and $120 \mathrm{mg} /$ day $(-11.0 ; p=0.004$ versus placebo) compared with placebo (-3.8) (Fig. 2). Results for completers (observed cases at day 42) are also shown in Fig. 2. Patients who completed treatment with lurasidone had numerically larger decreases in BPRSd scores than the overall LOCF analysis sample; however, there were no significant differences from placebo for either dose group for completers at day 42 , perhaps as a result of small sample size.

For the PANSS total score (secondary efficacy measure), the LS mean change from baseline to week 6 (LOCF) was significantly greater in the lurasidone $120 \mathrm{mg} /$ day group compared with placebo $(p=0.009)$, and a trend toward significance was noted for the lurasidone $40 \mathrm{mg} /$ day group $(p=0.076)$ (Table 2). Both lurasidone doses were significantly better than placebo with regard to improvement from baseline in PANSS positive symptoms score, CGI-S, and CGI-I. Patients receiving lurasidone $120 \mathrm{mg} /$ day (but not $40 \mathrm{mg} /$ day) also exhibited significantly greater improvement than those receiving placebo in PANSS negative symptoms and general psychopathology scores. Compared with the overall sample, study completers showed greater improvement with lurasidone (40 or $120 \mathrm{mg}$ /day) relative to placebo on each PANSS and CGI measure, but differences failed to reach statistical significance (data not shown).

The proportion of treatment responders based on a $\geq 20 \%$ decrease from baseline in BPRSd score at week 6 (LOCF) was significantly higher for the lurasidone $40(51.0 \%)$ and $120 \mathrm{mg} /$ day $(44.7 \%)$ treatment groups compared with the placebo group (18.4\%; $p<0.005$ for both comparisons) (Fig. 3). Similarly, a greater proportion of patients in the lurasidone $40(36.7 \%)$ and $120 \mathrm{mg} /$ day (30.4\%) groups had 
Fig. 1 Study flow diagram

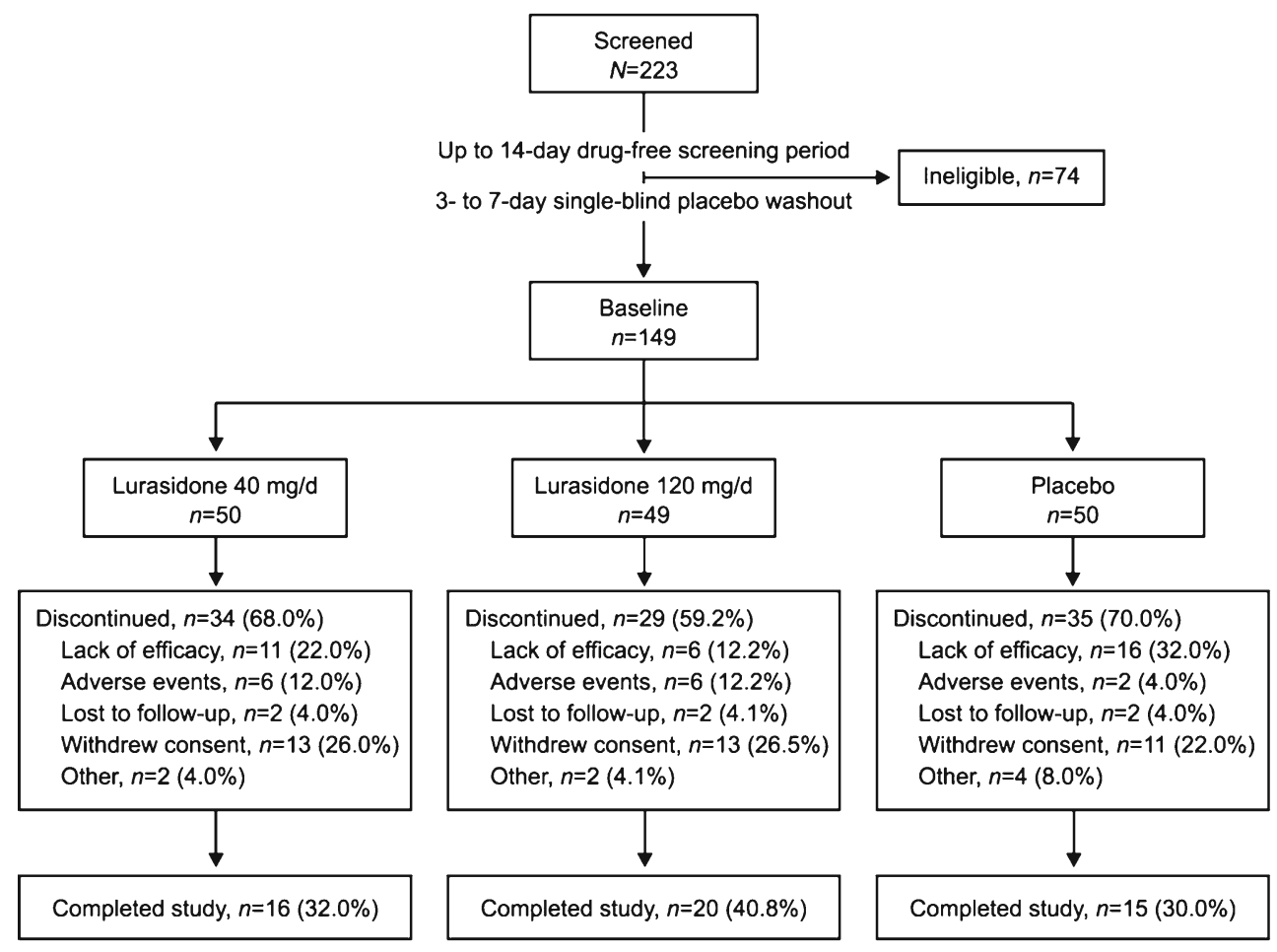

a CGI-I score of 1 or 2 (very much or much improved) at week 6 (LOCF) compared with patients in the placebo group (12.2\%; $<0.05$ for both comparisons). The effect size (LOCF) on the BPRSd was 0.53 for lurasidone $40 \mathrm{mg} /$ day and 0.65 for lurasidone $120 \mathrm{mg}$ /day. Effect sizes for secondary efficacy measures are shown in Table 2.

Table 1 Summary of patient characteristics at baseline (safety population)

\begin{tabular}{llll}
\hline Characteristic & Lurasidone $(40 \mathrm{mg} /$ day; $N=50)$ & Lurasidone $(120 \mathrm{mg} /$ day; $N=49)$ & Placebo $(N=50)$ \\
\hline Sex (male; $n(\%))$ & $36(72.0)$ & $36(73.5)$ & $42(84.0)$ \\
Race $(n(\%))$ & & & $22(44.9)$ \\
White & $20(40.0)$ & $24(49.0)$ & $25(50.0)$ \\
Black & $25(50.0)$ & $3(6.1)$ & $5(10.0)$ \\
Other & $5(10.0)$ & $41.0(9.0)$ & $38.1(9.7)$ \\
Age (years; mean (SD)) & $39.8(9.5)$ & $29.6(7.6)$ & $29.4(5.6)$ \\
Body mass index (kg/m ${ }^{2} ;$ mean (SD)) & $29.5(7.3)$ & & $45(90.0)$ \\
Schizophrenia subtype $(n(\%))$ & & $44(89.8)$ & $4(8.0)$ \\
Paranoid & $45(90.0)$ & $4(8.2)$ & $1(2.0)$ \\
Undifferentiated & $4(8.0)$ & $0(0.0)$ & $54.4(8.3)$ \\
Disorganized & $1(2.0)$ & & $53.5(39.0-74.0)$ \\
BPRSd & & $52.5(7.6)$ & $93.3(16.4)$ \\
Mean (SD) & $54.6(9.1)$ & $52.0(38.0-72.0)$ & $4.6(0.7)$ \\
Median (range) & $53.0(41.0-73.0)$ & $89.6(13.4)$ & $4.7(0.6)$ \\
PANSS total (mean (SD)) & $92.8(16.1)$ & $4.8(0.7)$ &
\end{tabular}

Differences among treatment groups for sex, race, and schizophrenia subtype were assessed using a Fisher exact test; differences for age, body mass index, BPRSd, PANSS, and CGI-S were assessed using analysis of variance with treatment in the model; differences in prior medications were not evaluated statistically. All available tests were not significant $(p>0.25)$

BPRSd Brief Psychiatric Rating Scale (derived from the PANSS), CGI-S Clinical Global Impression of Severity, PANSS Positive and Negative Syndrome Scale, $S D$ standard deviation 


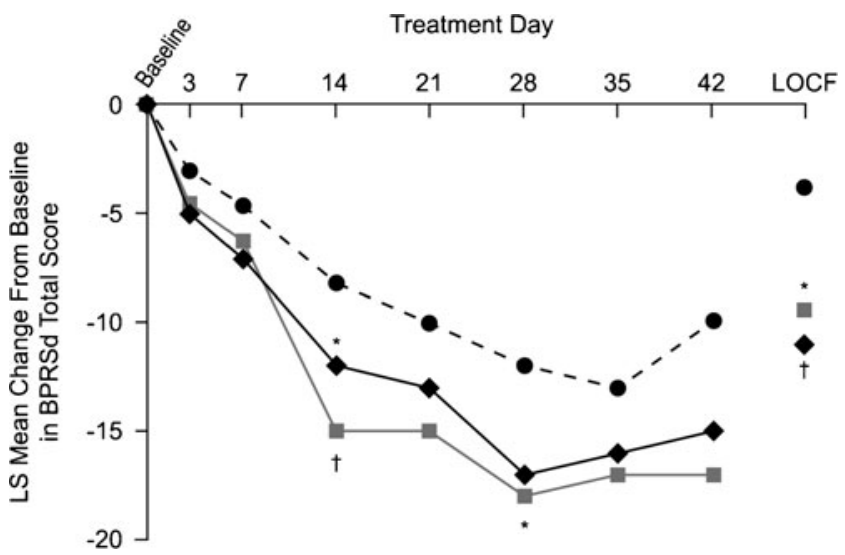

Fig. 2 Change from baseline in BPRSd score. Least-squares $(L S)$ mean change from baseline in BPRSd score. Analysis of covariance model with baseline value as covariate. Comparison with placebo based on a two-sided Dunnett $t$ test and based on last observation carried forward $(L O C F)$ analysis. ${ }^{*} p<0.05 ;{ }^{\dagger} p<0.01$. BPRSd Brief Psychiatric Rating Scale (derived from the PANSS), PANSS Positive and Negative Syndrome Scale. Circles, placebo, $n=49$ (LOCF), $n=17$ (day 42); squares, lurasidone $40 \mathrm{mg}, n=49$ (LOCF), $n=17$ (day 42); diamonds, lurasidone $120 \mathrm{mg}, n=47$ (LOCF), $n=19$ (day 42)

Tolerability and safety

\section{Adverse events}

The rate of adverse events occurring in $\geq 5 \%$ of patients is summarized in Table 3. The most commonly reported adverse events for patients receiving lurasidone were nausea (16.2\%), sedation (16.2\%), akathisia $(11.1 \%)$, dizziness $(11.1 \%)$, and headache (11.1\%). More patients receiving lurasidone $120 \mathrm{mg}$ /day reported nausea and akathisia (22.4 and $14.3 \%$, respectively) than those receiving lurasidone $40 \mathrm{mg}$ /day (10.0 and $8.0 \%$, respectively). The majority of adverse events were mild to moderate in intensity. The frequency of adverse events classified as severe was similar (6.0 $6.1 \%$ ) for the lurasidone and placebo groups.

An adverse event was cited as the reason for early treatment discontinuation for 14 patients: six $(12.0 \%)$ patients receiving lurasidone $40 \mathrm{mg} /$ day, six (12.2\%) patients receiving lurasidone $120 \mathrm{mg} / \mathrm{day}$, and two $(4.0 \%)$ patients receiving placebo. The most common adverse events leading to discontinuation were worsening of schizophrenia or other psychosis $(n=5)$, movement disorder symptoms $(n=3)$, and elevations in prolactin level $(n=2)$. Serious adverse events were reported for four patients $(8.0 \%)$ receiving lurasidone $40 \mathrm{mg} /$ day, two patients $(4.1 \%)$ receiving lurasidone $120 \mathrm{mg} /$ day, and four patients $(8.0 \%)$ receiving placebo, all of which were exacerbations of schizophrenia or other psychosis. No patients died during the study.

\section{Extrapyramidal symptoms}

Change from baseline to week 6 (LOCF) on measures of EPS and use of concomitant anticholinergic medication are shown in Table 4. There were no significant differences among treatment groups at any time point for mean SAS and AIMS scores. However, there were significant differences favoring lurasidone 40 compared with $120 \mathrm{mg}$ /day in mean BAS scores (change from baseline) at week 2 (lurasidone $40 \mathrm{mg} /$ day, -0.3 ; lurasidone $120 \mathrm{mg} /$ day, 1.4 ; and placebo, $-0.3 ; p=0.013$ ), week 4 (lurasidone $40 \mathrm{mg} / \mathrm{day}$, 0.0 ; lurasidone $120 \mathrm{mg} /$ day, 1.9 ; and placebo, $-0.6 ; p=$ 0.003 ), and week 5 (lurasidone $40 \mathrm{mg} / \mathrm{day},-0.4$; lurasidone $120 \mathrm{mg} /$ day, 1.0 ; and placebo, $-0.3 ; p=0.047$ ) but not at week 6 LOCF endpoint $(p=0.490)$. Movement disorder adverse events reported more frequently in patients receiving lurasidone 40 and $120 \mathrm{mg} /$ day versus placebo, respectively, included tremor (6.0 and 8.2 versus $0.0 \%$ ), muscle cramps (2.0 and 6.1 versus $0.0 \%$ ), extrapyramidal disorder (4.0 and 6.1 versus $0.0 \%$ ), and akathisia ( 8.0 and 14.3 versus $0.0 \%$ ). Three patients discontinued the study because of EPS or akathisia: one patient in the lurasidone $40 \mathrm{mg} /$ day group and two patients in the lurasidone $120 \mathrm{mg} /$ day group. The proportion of patients receiving concomitant benztropine for the treatment of movement disorders was higher in the lurasidone groups $(24.0 \%$ with $40 \mathrm{mg} /$ day and $24.5 \%$ with $120 \mathrm{mg} /$ day) than in the placebo group $(18.0 \%)$ (Table 4$)$.

\section{Body weight}

There were minimal changes in mean body weight in any treatment group at week 6 (LOCF) (Table 5).

\section{Metabolic and other laboratory tests}

Change in median total cholesterol from baseline to week 6 LOCF endpoint was comparable for patients treated with lurasidone $(-13 \mathrm{mg} / \mathrm{dL}$ for lurasidone $40 \mathrm{mg} /$ day and $-3 \mathrm{mg} / \mathrm{dL}$ for lurasidone $120 \mathrm{mg} /$ day) and patients in the placebo group $(-11.0 \mathrm{mg} / \mathrm{dL})$ (Table 5). Median triglyceride levels remained unchanged in the lurasidone $40 \mathrm{mg}$ /day group, increased by $16.5 \mathrm{mg} / \mathrm{dL}$ from baseline to week 6 LOCF endpoint in the lurasidone $120 \mathrm{mg}$ /day group, and decreased by $-11 \mathrm{mg} / \mathrm{dL}$ in the placebo group (Table 5). Median serum glucose levels were either unchanged or minimally decreased from baseline to week 6 LOCF endpoint in all groups (Table 5). There were no clinically significant hematology laboratory test results or urinalysis results reported. Clinically significant markedly abnormal chemistry values were observed in one patient taking lurasidone $120 \mathrm{mg} /$ day (elevated alanine aminotransferase (ALT), aspartate aminotransferase (AST), and triglycerides), 
Table 2 Change from baseline to week 6 (LOCF) in efficacy measures (intent-to-treat population)
Analysis of covariance models based on last observation carried forward with center and treatment as effects and baseline value as covariate

Comparison with placebo was performed using a two-sided, 0.050 Dunnett $t$ test

BPRSd Brief Psychiatric Rating Scale (derived from the PANSS), CGI-I Clinical Global Impression of Improvement, CGI-S Clinical Global Impression of Severity, $C I$ confidence interval, $L S$ least-squares, PANSS Positive and Negative Syndrome Scale, $S E$ standard error

\begin{tabular}{|c|c|c|c|}
\hline Efficacy measure & $\begin{array}{l}\text { Lurasidone (40 mg/day; } \\
N=49)\end{array}$ & $\begin{array}{l}\text { Lurasidone }(120 \mathrm{mg} / \text { day; } \\
N=47)\end{array}$ & Placebo $(N=49)$ \\
\hline \multicolumn{4}{|l|}{ BPRSd } \\
\hline $\begin{array}{l}\text { LS mean change (SE) } \\
\text { LS mean difference (SE) }\end{array}$ & $\begin{array}{l}-9.4(1.6) \\
-5.6(2.1)\end{array}$ & $\begin{array}{l}-11(1.6) \\
-6.7(2.2)\end{array}$ & \multirow[t]{3}{*}{$-3.8(1.6)$} \\
\hline Effect size & 0.53 & 0.65 & \\
\hline$p$ value & 0.018 & 0.004 & \\
\hline \multicolumn{4}{|l|}{ PANSS total score } \\
\hline $\begin{array}{l}\text { LS mean change (SE) } \\
\text { LS mean difference (SE) }\end{array}$ & $\begin{array}{l}-14(2.7) \\
-7.6(3.7)\end{array}$ & $\begin{array}{l}-17(2.7) \\
-11(3.7)\end{array}$ & \multirow[t]{3}{*}{$-6.2(2.7)$} \\
\hline Effect size & 0.42 & 0.60 & \\
\hline$p$ value & 0.076 & 0.009 & \\
\hline \multicolumn{4}{|l|}{ PANSS positive symptoms } \\
\hline $\begin{array}{l}\text { LS mean change (SE) } \\
\text { LS mean difference (SE) }\end{array}$ & $\begin{array}{l}-4.6(0.8) \\
-2.8(1.1)\end{array}$ & $\begin{array}{l}-5.1(0.8) \\
-3.3(1.1)\end{array}$ & \multirow[t]{3}{*}{$-1.8(0.8)$} \\
\hline Effect size & 0.53 & 0.63 & \\
\hline$p$ value & 0.018 & 0.005 & \\
\hline \multicolumn{4}{|l|}{ PANSS negative symptoms } \\
\hline $\begin{array}{l}\text { LS mean change (SE) } \\
\text { LS mean difference (SE) }\end{array}$ & $\begin{array}{l}-2.7(0.8) \\
-1.7(1.0)\end{array}$ & $\begin{array}{l}-4.0(0.8) \\
-2.9(1.1)\end{array}$ & \multirow[t]{3}{*}{$-1.0(0.8)$} \\
\hline Effect size & 0.33 & 0.56 & \\
\hline$p$ value & 0.177 & 0.011 & \\
\hline \multicolumn{4}{|c|}{ PANSS general psychopathology } \\
\hline $\begin{array}{l}\text { LS mean change (SE) } \\
\text { LS mean difference (SE) }\end{array}$ & $\begin{array}{l}-5.8(1.5) \\
-3.3(2.0)\end{array}$ & $\begin{array}{l}-7.8(1.5) \\
-5.3(2.1)\end{array}$ & \multirow[t]{3}{*}{$-2.5(1.5)$} \\
\hline Effect size & 0.33 & 0.53 & \\
\hline$p$ value & 0.185 & 0.023 & \\
\hline \multicolumn{4}{|l|}{ CGI-S } \\
\hline $\begin{array}{l}\text { LS mean change (SE) } \\
\text { LS mean difference (SE) }\end{array}$ & $\begin{array}{l}-0.8(0.2) \\
-0.7(0.2)\end{array}$ & $\begin{array}{l}-0.8(0.1) \\
-0.7(0.2)\end{array}$ & \multirow[t]{3}{*}{$-0.1(0.1)$} \\
\hline Effect size & 0.67 & 0.68 & \\
\hline$p$ value & 0.002 & 0.001 & \\
\hline \multicolumn{4}{|l|}{ CGI-I } \\
\hline $\begin{array}{l}\text { LS mean (SE) } \\
\text { LS mean difference (SE) }\end{array}$ & $\begin{array}{l}3.3(0.2) \\
-0.8(0.3)\end{array}$ & $\begin{array}{l}3.2(0.2) \\
-0.9(0.3)\end{array}$ & \multirow[t]{3}{*}{$4.1(0.2)$} \\
\hline Effect size & 0.62 & 0.68 & \\
\hline$p$ value & 0.006 & 0.002 & \\
\hline
\end{tabular}

and one patient in the placebo group (elevated ALT and AST). For one patient randomized to lurasidone $40 \mathrm{mg}$ /day, hyperglycemia of $3+$ glucosuria and elevated ALT were observed at baseline, prior to the first dose of study medication.

\section{Prolactin}

Median prolactin levels at week 6 LOCF endpoint were modestly increased relative to baseline in the lurasidone 40 $(3.5 \mathrm{ng} / \mathrm{mL})$ and $120 \mathrm{mg} /$ day $(7.7 \mathrm{ng} / \mathrm{mL})$ groups but not in the placebo group $(-1.3 \mathrm{ng} / \mathrm{mL})$ (Table 5$)$. A sex difference was observed, with lurasidone producing greater increases in prolactin in women than in men. Among women, the median change in prolactin at week 6 LOCF endpoint was $3.8 \mathrm{ng} / \mathrm{mL}$ in the lurasidone $40 \mathrm{mg} /$ day group, $21.1 \mathrm{ng} / \mathrm{mL}$ in the lurasidone $120 \mathrm{mg} /$ day group, and $0.4 \mathrm{ng} / \mathrm{mL}$ in the placebo group. Among men, smaller median changes in prolactin were observed in the lurasidone $40(3.4 \mathrm{ng} / \mathrm{mL})$ and $120 \mathrm{mg} /$ day $(5.6 \mathrm{ng} / \mathrm{mL})$ treatment groups; the median prolactin level in the placebo group decreased by $1.4 \mathrm{ng} / \mathrm{mL}$. Two patients (one female patient in the lurasidone $40 \mathrm{mg}$ / day group and one female patient in the lurasidone $120 \mathrm{mg} /$ day group) discontinued the study because of elevated prolactin based on predetermined elevations $(>200 \mathrm{ng} / \mathrm{mL})$. No prolactin-related clinical symptoms (e.g., galactorrhea) were observed. 


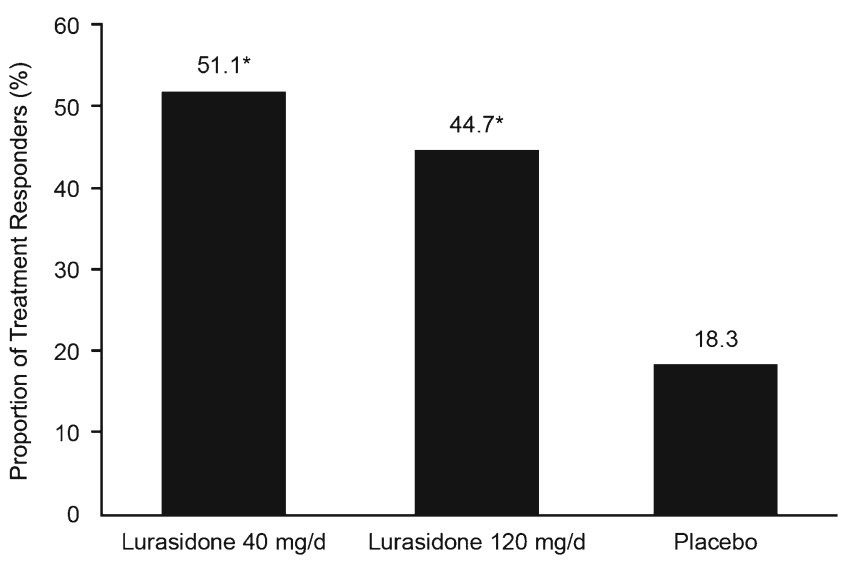

Fig. 3 Rates of treatment response. Proportion of patients classified as treatment responders based on a reduction of $\geq 20 \%$ from baseline to week 6 (LOCF) in BPRSd score. Lurasidone $40 \mathrm{mg} /$ day $(n=49)$; lurasidone $120 \mathrm{mg} /$ day $(n=47)$; and placebo $(n=49) .{ }^{*} p<0.01 \mathrm{com}-$ pared with placebo. BPRSd Brief Psychiatric Rating Scale (derived from the PANSS), LOCF last observation carried forward, PANSS Positive and Negative Syndrome Scale

\section{Physical examination and vital signs}

There were no clinically significant differences between lurasidone (40 or $120 \mathrm{mg} /$ day) and placebo in changes in vital signs at any assessment.

\section{Electrocardiogram}

Treatment with lurasidone was not associated with any clinically significant treatment-emergent ECG abnormalities. The results of ECG were comparable at all assessments. The mean QT interval corrected (QTc) was slightly decreased $(-3.3 \mathrm{~ms})$ in the lurasidone $120 \mathrm{mg} /$ day group and showed a small mean increase in both lurasidone $40 \mathrm{mg} /$ day $(3.0 \mathrm{~ms})$ and placebo (2.2 ms) groups (Table 5), but changes from baseline were not clinically meaningful. No patients in any of the three treatment groups had an increase in QTc interval of $>60 \mathrm{~ms}$. One patient, a 56-year-old woman in the placebo group, discontinued due to nonspecific ST-T segment ECG abnormalities.

\section{Discussion}

This was a phase 2 placebo-controlled study of lurasidone conducted in patients with schizophrenia and, as such, it included a limited sample size and was exploratory in nature. The study was not statistically powered to detect treatment effect sizes of the magnitude typically seen in placebocontrolled studies of atypical antipsychotic medications (Leucht et al. 2009b). Nonetheless, the results of this study
Table 3 Treatment-emergent adverse events occurring at a rate of $\geq 5 \%$ for any dose of lurasidone (safety population)

Comparison with placebo was performed using Fisher exact test

NOS not otherwise specified $* p<0.05$ versus placebo

\begin{tabular}{llll}
\hline Adverse event $(n(\%))$ & $\begin{array}{l}\text { Lurasidone } \\
(40 \mathrm{mg} / \text { day; } N=50)\end{array}$ & $\begin{array}{l}\text { Lurasidone } \\
(120 \mathrm{mg} / \text { day; } N=49)\end{array}$ & $\begin{array}{l}\text { Placebo } \\
(N=50)\end{array}$ \\
\hline Any adverse event & $40(80.0)$ & $38(77.6)$ & $36(72.0)$ \\
Nausea & $5(10.0)$ & $11(22.4)^{*}$ & $2(4.0)$ \\
Sedation & $9(18.0)$ & $7(14.3)$ & $5(10.0)$ \\
Headache NOS & $8(16.0)$ & $3(6.1)$ & $5(10.0)$ \\
Akathisia & $4(8.0)$ & $7(14.3)^{*}$ & $0(0)$ \\
Dizziness excluding vertigo & $6(12.0)$ & $5(10.2)$ & $3(6.0)$ \\
Dyspepsia & $4(8.0)$ & $2(4.1)$ & $6(12.0)$ \\
Somnolence & $4(8.0)$ & $5(10.2)$ & $2(4.0)$ \\
Vomiting NOS & $4(8.0)$ & $4(8.2)$ & $3(6.0)$ \\
Fatigue & $4(8.0)$ & $1(2.0)$ & $4(8.0)$ \\
Tremor & $3(6.0)$ & $4(8.2)$ & $0(0)$ \\
Insomnia & $3(6.0)$ & $4(8.2)$ & $0(0)$ \\
Diarrhea & $3(6.0)$ & $0(0)$ & $4(8.0)$ \\
Constipation & $4(8.0)$ & $0(0)$ & $3(6.0)$ \\
Back pain & $2(4.0)$ & $3(6.1)$ & $1(2.0)$ \\
Extrapyramidal disorder & $2(4.0)$ & $3(6.1)$ & $0(0)$ \\
Pain in limb & $3(6.0)$ & $1(2.0)$ & $1(2.0)$ \\
Muscle cramp & $1(2.0)$ & $3(6.1)$ & $0(0)$ \\
Any adverse event reported as severe & $3(6.0)$ & $3(6.1)$ & $3(6.0)$ \\
\hline
\end{tabular}


Table 4 Change in extrapyramidal symptom scores and use of concomitant anticholinergic medication (safety population)

\begin{tabular}{llll}
\hline & Lurasidone $(40 \mathrm{mg} /$ day; $N=50)$ & Lurasidone $(120 \mathrm{mg} /$ day; $N=49)$ & Placebo $(N=50)^{\mathrm{a}}$ \\
\hline Scale (mean (SD) change) & & & $-0.1(0.9)$ \\
SAS & $0.1(1.2)$ & $0.0(1.1)$ & $0.1(2.6)$ \\
BAS & $0.3(2.9)$ & $0.8(2.7)$ & $0.7(2.6)$ \\
AIMS & $0.6(2.8)$ & $0.3(2.4)$ & \\
Use of as-needed concomitant anticholinergic medication $(n(\%))$ & $12(24.5)$ & $(18.0)$ \\
Benztropine $^{\mathrm{b}}$ & $12(24.0)$ & & \\
\hline
\end{tabular}

Change from baseline in SAS, BAS, and AIMS was analyzed using one-way analysis of covariance

$S D$ standard deviation, SAS Simpson-Angus Scale, BAS Barnes Akathisia Scale, AIMS Abnormal Involuntary Movement Scale

${ }^{a}$ One to two missing values for scales

${ }^{\mathrm{b}}$ No other antiparkinson drugs were reported

indicate that lurasidone, at fixed daily doses of 40 and $120 \mathrm{mg}$ administered for 6 weeks, was an effective treatment for patients experiencing an acute exacerbation of chronic schizophrenia, with treatment effect sizes comparable to or greater than those in other clinical studies of atypical antipsychotics (Leucht et al. 2009b). Lurasidone 40 and $120 \mathrm{mg} /$ day produced significantly greater improvement than placebo on the primary efficacy measure, the change from baseline to week 6 (LOCF) in BPRSd score. On secondary efficacy measures, treatment with lurasidone 40 or $120 \mathrm{mg} /$ day resulted in significantly greater improvement than placebo in PANSS positive symptoms scores, CGI-S, and CGI-I. The $120 \mathrm{mg}$ /day dose also provided significant improvement in PANSS total, negative symptoms, and general psychopathology scores. The proportion of treatment responders was similar in the lurasidone 40 and $120 \mathrm{mg} /$ day groups and was significantly greater than in the placebo group.

Findings from this study have been replicated and extended in a larger $(N=478)$ phase 3 study with a similar patient population (Meltzer et al. 2011). This 6-week, randomized, double-blind, placebo-controlled study also included olanzapine as an active comparator to confirm assay sensitivity.
Table 5 Change from baseline to week 6 (LOCF) in key safety parameters
When possible, blood samples for evaluation of lipid, glucose, and prolactin levels were collected with patients in the fasted state

QTc interval QT interval corrected, $S D$ standard deviation

${ }^{\mathrm{a}} n=48$

$\mathrm{b}_{n=49}$

${ }^{\mathrm{c}} n=41$

${ }^{\mathrm{d}} n=40$

\begin{tabular}{|c|c|c|c|}
\hline Safety parameter & $\begin{array}{l}\text { Lurasidone } \\
(40 \mathrm{mg} / \text { day; } N=50)\end{array}$ & $\begin{array}{l}\text { Lurasidone } \\
(120 \mathrm{mg} / \text { day; } N=49)\end{array}$ & Placebo $(N=50)$ \\
\hline \multicolumn{4}{|l|}{ Weight (kg) } \\
\hline Mean (SD) baseline value & $87.4(22.0)^{\mathrm{a}}$ & $90.2(25.7)^{\mathrm{b}}$ & $89.3(20.2)^{\mathrm{b}}$ \\
\hline Mean (SD) change & $0.3(2.2)$ & $0.2(2.6)$ & $0.0(2.9)$ \\
\hline \multicolumn{4}{|l|}{ Total cholesterol (mg/dL) } \\
\hline Mean (SD) baseline value & $212(45.8)^{\mathrm{c}}$ & $194(62.8)^{\mathrm{d}}$ & $219(38.9)^{\mathrm{c}}$ \\
\hline Median change & -13.0 & -3.0 & -11.0 \\
\hline \multicolumn{4}{|l|}{ Triglycerides (mg/dL) } \\
\hline Mean (SD) baseline value & $190(137)^{\mathrm{c}}$ & $272(379)^{\mathrm{d}}$ & $275(187)^{\mathrm{c}}$ \\
\hline Median change & 0.0 & 16.5 & -11.0 \\
\hline \multicolumn{4}{|l|}{ Serum glucose (mg/dL) } \\
\hline Mean (SD) baseline value & $105(42.0)^{\mathrm{c}}$ & $113(46.8)^{\mathrm{d}}$ & $98.1(19.2)^{\mathrm{c}}$ \\
\hline Median change & 0.0 & -2.0 & -0.5 \\
\hline \multicolumn{4}{|l|}{ Prolactin (ng/mL) } \\
\hline Mean (SD) baseline value & $9.0(4.7)^{\mathrm{c}}$ & $10.8(8.0)^{\mathrm{d}}$ & $14.8(23.7)^{\mathrm{c}}$ \\
\hline Median change & 3.5 & 7.7 & -1.3 \\
\hline \multicolumn{4}{|l|}{ QTc interval } \\
\hline Mean (SD) baseline value (ms) & $421.2(20.0)$ & $420.8(24.5)^{\mathrm{a}}$ & $416.8(20.0)$ \\
\hline Mean (SD) change (ms) & $3.0(23.6)$ & $-3.3(19.9)$ & $2.2(21.2)$ \\
\hline Increase of $>60 \mathrm{~ms}(n(\%))$ & $0(0)$ & $0(0)$ & $0(0)$ \\
\hline
\end{tabular}


Results demonstrated that lurasidone 40 and $120 \mathrm{mg} /$ day produced significantly greater improvement than placebo in PANSS total score (the primary outcome measure), PANSS subscale scores, and CGI-S score (Meltzer et al. 2011). Based on a post-hoc analysis, there were no significant differences in efficacy for lurasidone versus olanzapine (Meltzer et al. 2011).

In the present study, lurasidone $120 \mathrm{mg} /$ day significantly improved PANSS total score (secondary measure) compared with placebo $(p=0.009)$, and a trend toward significance was observed for lurasidone $40 \mathrm{mg} /$ day $(p=0.076)$. The ability to detect a significant effect for lurasidone $40 \mathrm{mg} /$ day on PANSS total score may have been limited by the relatively small sample size in this study.

The efficacy of lurasidone in the treatment of schizophrenia was also demonstrated in a study that evaluated an intermediate dose, $80 \mathrm{mg} / \mathrm{day}$, for the treatment of schizophrenia (Nakamura et al. 2009). In that 6-week, randomized, doubleblind, placebo-controlled study $(N=180)$, patients receiving lurasidone $80 \mathrm{mg} /$ day showed significantly greater improvement from baseline to week 6 (LOCF) in BPRSd (primary measure) than patients receiving placebo (Nakamura et al. 2009). Lurasidone $80 \mathrm{mg}$ /day also produced significantly greater improvement than placebo on secondary measures, including the PANSS total score, PANSS subscale scores (positive, negative, cognitive, and general psychopathology subscales), and CGI-S (Nakamura et al. 2009).

Metabolic adverse effects associated with atypical antipsychotic therapy, including weight gain and changes in lipid and glucose levels, are a cause of substantial concern. Treatment with atypical antipsychotic medications has been linked to increased risk of obesity, diabetes, and dyslipidemia for patients with schizophrenia (American Diabetes Association 2004). According to a meta-analysis including head-to-head comparisons of nine atypical antipsychotic medications (amisulpride, aripiprazole, clozapine, olanzapine, quetiapine, risperidone, sertindole, ziprasidone, and zotepine), some agents were associated with considerably greater weight gain and metabolic adverse effects than others (Rummel-Kluge et al. 2010). Specifically, clozapine and olanzapine produced the greatest elevations in weight, cholesterol, and glucose. Quetiapine, risperidone, and sertindole showed intermediate elevations in these metabolic parameters. Aripiprazole, amisulpride, and ziprasidone were associated with small changes in weight and metabolic indices (Rummel-Kluge et al. 2010).

An important finding of the current study is that lurasidone had minimal effects on body weight and other metabolic parameters. Mean weight change from baseline was small and consistent with other studies of lurasidone (Meltzer et al. 2011; Nakamura et al. 2009). Other metabolic indices, including cholesterol, triglyceride, and glucose levels, either decreased or remained essentially unchanged, as has been reported in other studies of lurasidone (Meltzer et al. 2011;
Nakamura et al. 2009; Potkin et al. 2011). Together, these findings suggest that the clinically significant weight gain and metabolic changes observed during short-term treatment with some atypical antipsychotics (Leucht et al. 2009a) are unlikely to occur with lurasidone. Lurasidone's low propensity for weight gain and minimal effect on glucose and lipid parameters may be an important treatment consideration, especially in light of the increased rate of metabolic syndrome among patients with schizophrenia (Correll et al. 2010; Newcomer 2007). Lurasidone may be a particularly appropriate treatment for patients who are concerned about the potential for weight gain, dyslipidemia, and hyperglycemia during antipsychotic treatment.

Adverse events observed in the present study were generally comparable with those that have been previously reported in other studies of lurasidone (Meltzer et al. 2011; Nakamura et al. 2009). Most adverse events were rated as mild to moderate in intensity. The proportion of patients who experienced adverse events rated as severe was low and comparable for lurasidone and placebo (6.0-6.1\%).

Lurasidone appeared to be better tolerated at 40 than $120 \mathrm{mg} /$ day in the present study. Nausea was more common among patients treated with lurasidone $120 \mathrm{mg}$ /day compared with $40 \mathrm{mg} /$ day. Although results of this study suggest that treatment with lurasidone at doses of 40 and $120 \mathrm{mg} /$ day has a low propensity for causing EPS, the occurrence of akathisia appeared to be dose related. Treatment with lurasidone resulted in modest, dose-dependent increases in prolactin levels, which were more pronounced in female patients. Similar increases in prolactin were observed with lurasidone treatment in other short-term studies (Meltzer et al. 2011; Nakamura et al. 2009; Potkin et al. 2011). The mean increase in prolactin levels observed with lurasidone $120 \mathrm{mg} /$ day was lower than that reported following shortterm treatment with risperidone, paliperidone, or conventional antipsychotics (Bostwick et al. 2009). No galactorrhea or other clinically important prolactin-related adverse events were reported in this study.

Limitations of the current study include the short duration, small sample size, and high rate of discontinuation, which make it difficult to draw firm conclusions about the treatment effects of lurasidone. The all-cause discontinuation rate was 59 to $68 \%$ for patients treated with lurasidone and $70 \%$ in the placebo group. The average all-cause discontinuation rate in short-term (4- to 12-week) placebo-controlled studies of atypical antipsychotics is $48 \%$ for active treatment and $60 \%$ for placebo (Kemmler et al. 2005). In contrast to the present study, other studies of lurasidone had lower-than-average rates of discontinuation (Meltzer et al. 2011; Nakamura et al. 2009). All-cause discontinuation rates in a larger 6-week study of lurasidone 40 and $120 \mathrm{mg} /$ day were 36 and $45 \%$, respectively, compared with $39 \%$ for placebo (Meltzer et al. 2011). In the present study, the protocol required that patients must be 
discontinued from study participation if they had not improved enough by week 4 to permit hospital discharge, which may have contributed to the relatively high rates of discontinuation due to lack of efficacy. Since this was an early study of a novel medication, investigators may have had concerns about the efficacy and tolerability of the investigational medication, leading to higher discontinuation rates than observed in subsequent studies and increased use of rescue medications. Supporting this view, use of benzodiazepines occurred at similar high rates in the lurasidone and placebo groups.

In conclusion, this study, which was limited by a relatively high rate of discontinuation, suggests that short-term administration of lurasidone in doses of 40 or $120 \mathrm{mg} /$ day was effective in the treatment of patients experiencing an acute exacerbation of chronic schizophrenia. Treatment with lurasidone had minimal effects on weight and metabolic parameters. Lurasidone may offer an effective once-daily treatment with few metabolic complications for patients with schizophrenia.

Acknowledgments and disclosures Masaaki Ogasa and Tatsuya Kimura are full-time employees of Dainippon Sumitomo Pharma Co., Ltd. Mitsutaka Nakamura was an employee of Dainippon Sumitomo Pharma Co., Ltd. at the time the study was conducted, but he is currently employed at Setsunan University, Osaka, Japan. John Guarino is an employee of Sunovion Pharmaceuticals, Inc. This study was sponsored by Dainippon Sumitomo Pharma Co., Ltd., Osaka, Japan. Technical medical writing and editorial assistance was provided by Adrienne Drinkwater, PhD, for Synchrony Medical, LLC, West Chester, PA. Funding for this support was provided by Sunovion Pharmaceuticals, Inc., Marlborough, MA. The authors would like to thank the patients who participated in this study, as well as the study investigators: A. Adityanjee, M. Bari, B. Bastani, R. Bloch, Stanley Cheren, A.J. Cutler, R. Gopalan, C. Goldman, M. Plopper, R. Riesenberg, T.K. Tran-Johnson, R. Wang, E. Logue, M. Rosenthal, and M.Y. Alam.

Previous presentations This study was presented, in part, at the Annual Meeting of the American Psychiatric Association, 16-21 May 2009, San Francisco, CA, and the Annual Meeting of the New Clinical Drug Evaluation Unit, 29 June-2 July 2009, Hollywood, FL.

Open Access This article is distributed under the terms of the Creative Commons Attribution License which permits any use, distribution, and reproduction in any medium, provided the original author(s) and the source are credited.

\section{References}

American Diabetes Association, American Psychiatric Association, American Association of Clinical Endocrinologists, North American Association for the Study of Obesity (2004) Consensus development conference on antipsychotic drugs and obesity and diabetes. J Clin Psychiatry 65:267-272

Barnes TR (1989) A rating scale for drug-induced akathisia. Br J Psychiatry 154:672-676

Bostwick JR, Guthrie SK, Ellingrod VL (2009) Antipsychotic-induced hyperprolactinemia. Pharmacotherapy 29:64-73
Correll CU, Druss BG, Lombardo I, O’Gorman C, Harnett JP, Sanders KN, Alvir JM, Cuffel BJ (2010) Findings of a U.S. national cardiometabolic screening program among 10,084 psychiatric outpatients. Psychiatr Serv 61:892-898

First MB, Spitzer RL, Gibbon M, Williams JBW (1997) Structured clinical interview for DSM-IV (SCID). American Psychiatric Press, Washington, DC

Guy W (ed.) (1976) ECDEU assessment manual for psychopharmacology, revised. US Department of Health, Education, and Welfare Pub. No. (ADM) 76-338. National Institute of Mental Health, Rockville, MD

Henderson DC, Cagliero E, Copeland PM, Borba CP, Evins E, Hayden D, Weber MT, Anderson EJ, Allison DB, Daley TB, Schoenfeld D, Goff DC (2005) Glucose metabolism in patients with schizophrenia treated with atypical antipsychotic agents: a frequently sampled intravenous glucose tolerance test and minimal model analysis. Arch Gen Psychiatry 62:19-28

International Conference on Harmonisation (ICH) (1996) ICH harmonised tripartite guideline: guideline for good clinical practice. Available at http://www.ich.org/fileadmin/Public Web Site/ ICH_Products/Guidelines/Efficacy/E6_R1/Step4/E6_R1 Guideline.pdf. Accessed 17 Jan 2012

Ishibashi T, Horisawa T, Tokuda K, Ishiyama T, Ogasa M, Tagashira R, Matsumoto K, Nishikawa H, Ueda Y, Toma S, Oki H, Tanno N, Saji I, Ito A, Ohno Y, Nakamura M (2010) Pharmacological profile of lurasidone, a novel antipsychotic agent with potent 5hydroxytryptamine $7\left(5-\mathrm{HT}_{7}\right)$ and $5-\mathrm{HT}_{1 \mathrm{~A}}$ receptor activity. J Pharmacol Exp Ther 334:171-181

Kay SR, Fiszbein A, Opler L (1987) The Positive and Negative Syndrome Scale (PANSS) for schizophrenia. Schizophr Bull 13:261-276

Kemmler G, Hummer M, Widschwendter C, Fleischhacker WW (2005) Dropout rates in placebo-controlled and active-control clinical trials of antipsychotic drugs: a meta-analysis. Arch Gen Psych 62:1305-1312

Kroeze WK, Hufeisen SJ, Popadak BA, Renock SM, Steinberg S, Ernsberger P, Jayathilake K, Meltzer HY, Roth BL (2003) H1histamine receptor affinity predicts short-term weight gain for typical and atypical antipsychotic drugs. Neuropsychopharmacology 28:519-526

Latuda ${ }^{\circledR}$ (lurasidone $\mathrm{HCl}$ ) Tablets US prescribing information. http:// www.latuda.com/LatudaPrescribingInformation.pdf. Accessed 28 October 2010.

Leucht S, Corves C, Arbter D, Engel RR, Li C, Davis JM (2009a) Second-generation versus first-generation antipsychotic drugs for schizophrenia: a meta-analysis. Lancet 373:31-41

Leucht S, Arbter D, Engel RR, Kissling W, Davis JM (2009b) How effective are second-generation antipsychotic drugs? A metaanalysis of placebo-controlled trials. Mol Psychiatry 14:429-447

Lieberman JA, Stroup TS, McEvoy JP, Swartz MS, Rosenheck RA, Perkins DO, Keefe RS, Davis SM, Davis CE, Lebowitz BD, Severe J, Hsiao JK, Clinical Antipsychotic Trials of Intervention Effectiveness (CATIE) Investigators (2005) Effectiveness of antipsychotic drugs in patients with chronic schizophrenia. N Engl J Med 353:1209-1223

Lindenmayer J-P, Grochowski S, Hyman RB (1995) Five factor model of schizophrenia: replication across samples. Schizophr Res 14:229-234

Meltzer HY, Cucchiaro J, Silva R, Ogasa M, Phillips D, Xu J, Kalali AH, Schweizer E, Pikalov A, Loebel A (2011) Lurasidone in the treatment of schizophrenia: a randomized, double-blind, placebo and olanzapine-controlled study. Am J Psychiatry 168:957-967

Meltzer HY, Li Z, Kaneda Y, Ichikawa J (2003) Serotonin receptors: their key role in drugs to treat schizophrenia. Prog Neuropsychopharmacol Biol Psychiatry 27:1159-1172

Nakamura M, Ogasa M, Guarino J, Phillips D, Severs J, Cucchiaro J, Loebel A (2009) Lurasidone in the treatment of acute schizophrenia: a double-blind, placebo-controlled trial. J Clin Psychiatry 70:829 836 
Newcomer JW (2007) Antipsychotic medications: metabolic and cardiovascular risk. J Clin Psychiatry 68(suppl 4):8-13

Potkin SG, Ogasa M, Cucchiaro J, Loebel A (2011) Double-blind comparison of the safety and efficacy of lurasidone and ziprasidone in clinically stable outpatients with schizophrenia or schizoaffective disorder. Schizophr Res 132:101-107

Regier DA, Narrow WE, Rae DS, Manderscheid RW, Locke BZ, Goodwin FK (1993) The de facto US mental and addictive disorders service system. Epidemiologic catchment area prospective 1 -year prevalence rates of disorders and services. Arch Gen Psychiatry 50:85-94

Rummel-Kluge C, Komossa K, Schwarz S, Hunger H, Schmid F, Lobos CA, Kissling W, Davis JM, Leucht S (2010) Head-tohead comparisons of metabolic side effects of second generation antipsychotics in the treatment of schizophrenia: a systematic review and meta-analysis. Schizophr Res 123:225-233

Schotte A, Janssen PF, Gommeren W, Luyten WH, Van Gompel P, Lesage AS, De Loore K, Leysen JE (1996) Risperidone compared with new and reference antipsychotic drugs: in vitro and in vivo receptor binding. Psychopharmacol (Berl) 124:57-73

Sernyak MJ, Rosenheck RA (2008) Antipsychotic use in the treatment of outpatients with schizophrenia in the VA from fiscal years 1999 to 2006. Psychiatr Serv 59:567-569

Simpson GM, Angus JW (1970) A rating scale for extrapyramidal side effects. Acta Psychiatr Scand Suppl 212:11-19

Wu EQ, Birnbaum HG, Shi L, Ball DE, Kessler RC, Moulis M, Aggarwal J (2005) The economic burden of schizophrenia in the United States in 2002. J Clin Psychiatry 66:1122-1129 